\title{
CART cells targeting CD99 as an approach to eradicate T-cell acute lymphoblastic leukemia without normal blood cells toxicity
}

\author{
Jiangzhou Shi ${ }^{1 \dagger}$, Zijian Zhang ${ }^{1 \dagger}$, Hong $\mathrm{Cen}^{2 \dagger}{ }^{\dagger}$, Han Wu$^{1}$, Shangkun Zhang ${ }^{1}$, Jiaxing Liu' ${ }^{1}$, Yingqi Leng ${ }^{1}$, Anqi Ren ${ }^{1}$, \\ Xiyu Liu' ${ }^{1}$, Zhijie Zhang ${ }^{1}$, Xiqin Tong ${ }^{3}$, Jinjue Liang ${ }^{1}$, Zhe Li ${ }^{2}$, Fuling Zhou ${ }^{3}$, Liang Huang ${ }^{4}$, You Qin ${ }^{5}$, Kunyu Yang ${ }^{5}$, \\ Tongcun Zhang ${ }^{1,6^{*}}$ and Haichuan Zhu ${ }^{1 *}$ (D)
}

\begin{abstract}
CART cell therapy has shown dramatic clinical success in relapsed or refractory B-ALL and other hematological malignancies. However, the loss of specific antigens, cell fratricide, T cell aplasia, and normal T cell separation are challenges in treating T cell leukemia/lymphoma with CART therapy. CD99 is a promising antigen to target T-ALL and AML as it is strongly expressed on the majority of T-ALL and AML. Here, we isolated a low-affinity CD99 (12E7) antibody, which specifically recognizes leukemia cells over normal blood cells. Moreover, T cells transduced with an anti-CD99specific CAR that contained the 12E7 scFv expanded with minor fratricide and without normal blood cells toxicity. We observed that our anti-CD99 CART cells showed robust cytotoxicity specifically against CD99+ T-ALL cell lines and primary tumor cells in vitro and significantly prolonged cell line-derived xenografts (CDXs) or patient-derived xenografts (PDXs) models survival in vivo. Together, our results demonstrate that anti-CD99 CART cells could specifically recognize and efficiently eliminate CD99+ leukemia cells.
\end{abstract}

Keywords: CART, CD99, T-ALL, AML, Antitumor activity

\section{To the editor:}

T-ALL is an aggressive hematological malignancy accounting for $15 \%$ of pediatric and $25 \%$ of adult ALL cases $[1,2]$. The standard treatment of chemotherapy combined with glucocorticoids has significantly improved survival, but up to $20 \%$ of pediatric and $40 \%$ of adult T-ALL patients are at risk for relapse [3, 4]. Novel optimal therapeutic strategies need to be developed for T-ALL, particularly for relapsed and refractory T-ALL patients. CARs targeting CD19 have been studied extensively for the treatment of B-ALL $[5,6]$. However, CAR

\footnotetext{
*Correspondence: zhangtongcun@wust.edu.cn; zhuhaichuan@wust.edu.cn ${ }^{\dagger}$ Jiangzhou Shi, Zijian Zhang and Hong Cen have contributed equally to this work

${ }^{1}$ Institute of Biology and Medicine, College of Life and Health Sciences, Wuhan University of Science and Technology, Wuhan 430081, China

Full list of author information is available at the end of the article
}

$\mathrm{T}$ cells share similar antigens with malignant $\mathrm{T}$ cells, and translating this approach to T-ALL has been extremely challenging due to fratricide and $\mathrm{T}$ cell aplasia [7].

CD99 has been demonstrated to have stronger expression in newly diagnosed T-ALL and also used as a new tool for the detection of MRD [8, 9]. We also confirmed CD99 up-regulated in transcript and protein levels compared with normal $\mathrm{T}$ cells (Additional file 1: Fig S1a, b). Further, we found that the CD99 expression is strong in different subgroups with the meanings of FPKM above 100 (Additional file 1: Fig S1c, d), indicating that CAR $\mathrm{T}$ cell therapy based on CD99 is a promising therapeutic strategy for T-ALL rooting out. To avoid T cell fratricide and potential on-target, off-tumor effects, we first

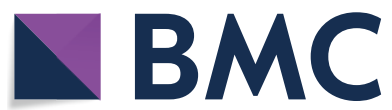

(c) The Author(s) 2021. Open Access This article is licensed under a Creative Commons Attribution 4.0 International License, which permits use, sharing, adaptation, distribution and reproduction in any medium or format, as long as you give appropriate credit to the original author(s) and the source, provide a link to the Creative Commons licence, and indicate if changes were made. The images or other third party material in this article are included in the article's Creative Commons licence, unless indicated otherwise in a credit line to the material. If material is not included in the article's Creative Commons licence and your intended use is not permitted by statutory regulation or exceeds the permitted use, you will need to obtain permission directly from the copyright holder. To view a copy of this licence, visit http://creativecommons.org/licenses/by/4.0/. The Creative Commons Public Domain Dedication waiver (http://creativeco mmons.org/publicdomain/zero/1.0/) applies to the data made available in this article, unless otherwise stated in a credit line to the data. 
identified a lower affinity anti-CD99 mAb (12E7) that specifically recognizes CD99-expressing T-ALL cell lines but not normal blood cells (Fig. 1a). Then, we systematically investigated the sensitivity of $12 \mathrm{E} 7 \mathrm{mAb}$ in normal tissue and observed 12E7 mAb-positive signals only in parts of the thymus, but not in the spleen, liver, kidney or other important organs (Additional file 1: Fig S1e). We also found the 12E7 scFv exhibited a lower binding affinity of $6.97 \times 10^{-8} \mathrm{M}$, which is lower than the 1021527 $\left(5.76 \times 10^{-9} \mathrm{M}\right)$ and $3 \mathrm{~B} 2 / \mathrm{TA} 8\left(1.93 \times 10^{-9} \mathrm{M}\right)($ Fig. $1 \mathrm{~b}$ and Additional file 2: Fig S1f). And there was a strong positive correlation between the $12 \mathrm{E} 7 \mathrm{mAb}$ and $12 \mathrm{E} 7 \mathrm{scFv}$ in the different cell lines according to flow cytometry analysis (Fig. 1c). Together, the results indicate that the 12E7 mAb is an optimal antibody for anti-CD99 CAR $\mathrm{T}$ therapy according to its specific target molecule recognition and limited binding to normal cells.

Next, the 12E7 scFv was incorporated into the lentivirus CAR vector to generate anti-CD99 CAR (Fig. 1d). Following activation and transduction of T cells, anti-CD99 CAR T cells were significantly fewer than the anti-CD19 CAR $\mathrm{T}$ control (Fig. 1e). Interestingly, the efficacy of transduced CAR+ cells was significantly increased during cell culture, almost $100 \%$ in the 12 days (Fig. 1f). And the following results showed that CD99 induced expression after CD3/CD28 beads activation and could be targeted by anti-CD99 CAR T cells (Fig. 1g, h). In contrast, we purified the CAR + cells after 3 days transduction and found the antigen of CD99 did not express in anti-CD99 CAR T cells and CAR+ cells could not exhibit significant fratricide during the cell culture (Fig. 1i, j). Next, we assessed the antigen specificity and cytotoxic activity of anti-CD99 CAR T cells in NIH 3T3 human CD99 overexpression cell line and MOLT-4 CD99 knockdown cell line and found that the cytotoxicity was strongly correlated with the expression level of CD99 (Additional file 2:
Fig. S1g-h). Furthermore, anti-CD99 CAR T cells showed specifically target the T-ALL cell lines and primary cells but with minimal killing of normal blood cells (Fig. 1k-m and Additional file 2: Fig. S1i). In addition, not only the T-ALL, we also found CD99 expressed and induced powerful antitumor activity in AML and a vast majority of solid tumor cells (Additional file 3: Fig. S1j, k), suggesting that CD99 may be a broad spectrum target for CAR T cell therapy.

To assess the effect of anti-CD99 CAR T cells against CD99+ T-ALL cells in vivo, we performed experiments using CDXs and PDXs models created by Jurkat, MOLT4, and patient samples, respectively (Fig. 2a). Anti-CD99 CAR T cells conferred robust protection against leukemia progression and significantly extended the median survival of the mice in the CDX and PDX models (Fig. 2b-g). Especially in the PDX-1 model, the engraftment of CD99+ primary T-ALL cells gradually increased over time in the $\mathrm{PB}$ of the control $\mathrm{T}$ cell groups, whereas anti-CD99 CAR T cells significantly delayed leukemia progression (Fig. 2c). Compared with the $\mathrm{T}$ cell treatment group, anti-CD99 CAR T cells significantly eliminated infiltrating leukemia cells in the spleen and BM (Fig. 2h, $i$ and Additional file 4: Fig. S2a-e). More importantly, the results showed that CARs persisted at a detectable level in the PB for at least 21 days in the Jurkat CDX model treated with CAR $T$ cells (Fig. 2j) but without a significant change in animal body weight (Fig. 2k).

In summary, we demonstrated that CD99 is an attractive target for the immunotherapy of T-ALL and provided preclinical evidence for the therapeutic and safe use of fratricide-resistant anti-CD99 CAR T cells. Importantly, future clinical trials will need to assess the safety and feasibility of anti-CD99 CAR $\mathrm{T}$ cell therapy.

\footnotetext{
(See figure on next page.)

Fig. 1 a CD99 recognizing ability of three anti-CD99 mAbs (12E7, 1021527 and 3B2/TA8) in normal blood cells and leukemia cell lines (Jurkat and MOLT-4) by flow cytometry. $\mathbf{b}$ Binding kinetics of anti-CD99 scFv with CD99 protein. Analysis of the interaction between the 12 E7 scFv and CD99 protein using BLItz biolayer interferometry. c The 12E7 antibody and the anti-CD99 scFv from $12 \mathrm{E} 7$ showed a strong correlation in different cell lines based on MFI analysis. d Schematic illustration of the anti-CD99 CAR construct. e Expansion fold change of total T cells transduced with CD19 CAR or CD99 CAR for 14 days. f Percentage of CAR positive cells measured by flow cytometry using Strep-Tag $\|$ antibody during the CAR T cells in vitro culture. $\mathbf{g}$ Expression of CD99 in normal T cells activated by CD3/CD28 beads. $\mathbf{h}$ Cytotoxic activity of anti-CD99 CART cells against normal T cells which activated by CD3/CD28 beads in different days determined by calcein release assay at the ratios of 25:1 after 2-3 h co-culture. $\mathbf{i}$ Expression of CD99 in CAR positive cells by flow cytometry. $\mathbf{j}$ Expansion fold change of CAR positive cells for 12 days. $\mathbf{k}$ In vitro cytotoxic activity of anti-CD99 CART cells against different normal blood cells. I and $\mathbf{m}$ Cytotoxic activity of anti-CD99 CART cells against T-ALL cell lines (Jurkat/ CUTLL-1) and T-ALL patients' blasts (Patient \#1/ Patient \#2/ Patient \#3/ Patient \#4) as determined by calcein release assay at different E:T ratios (5:1,25:1) after 2-3 h co-culture. IgG as the negative control.*** $p \leq 0.001,{ }^{* *} p \leq 0.01, \mathrm{NS}=$ no significant
} 


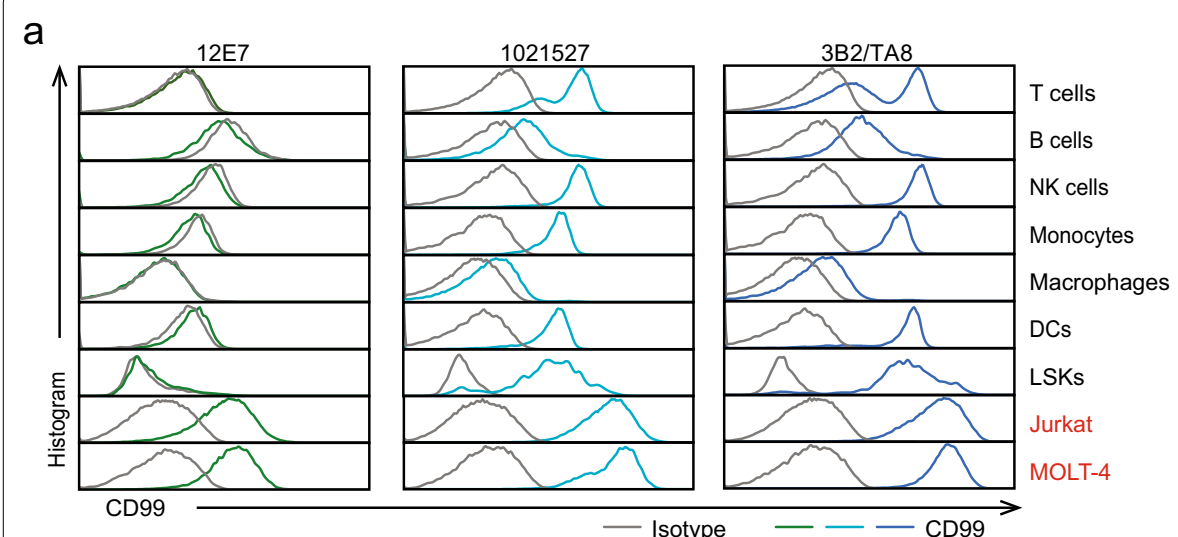

d
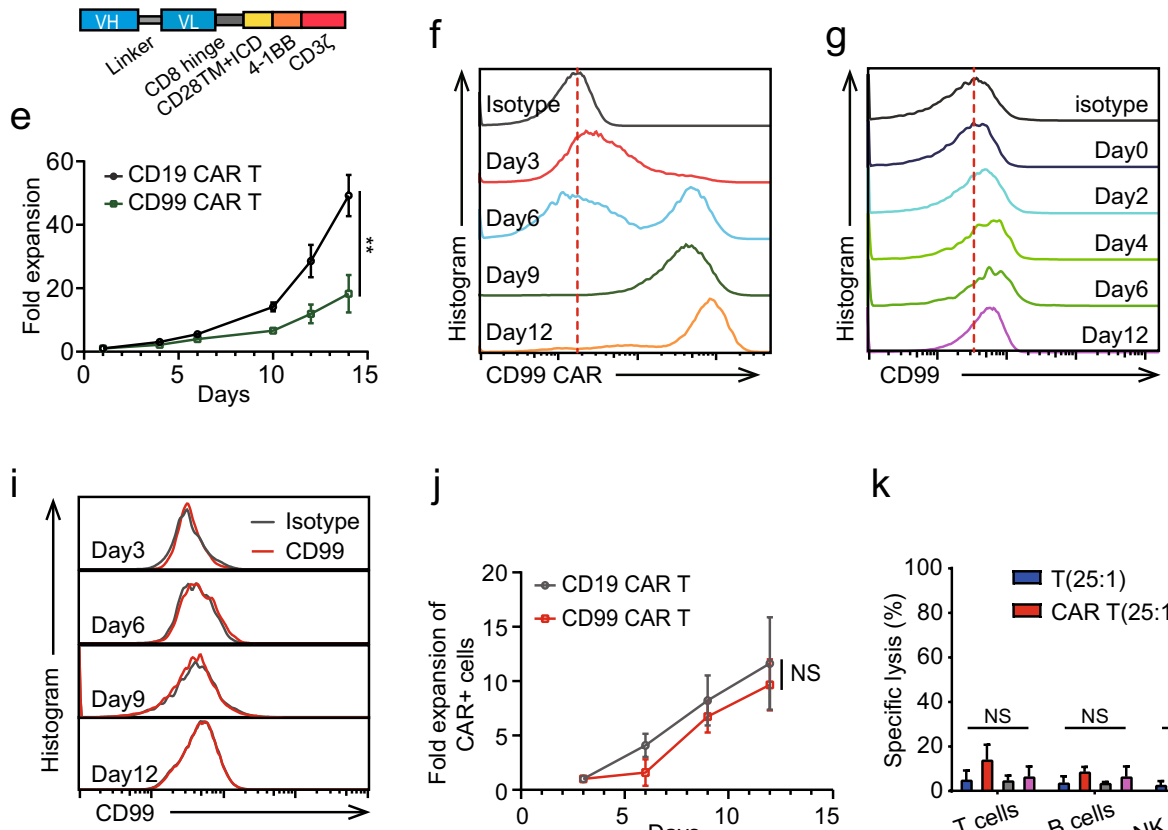

k b

C
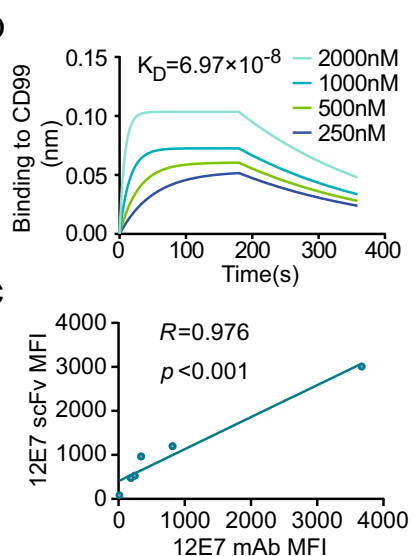

h
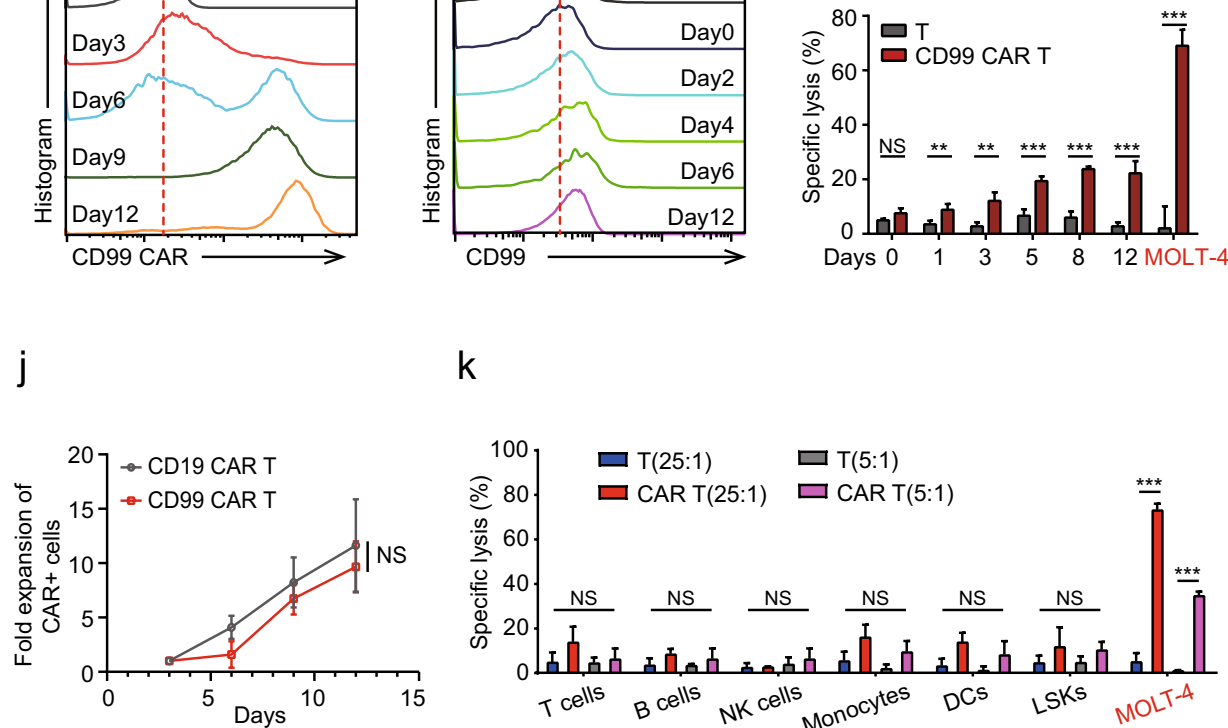

I

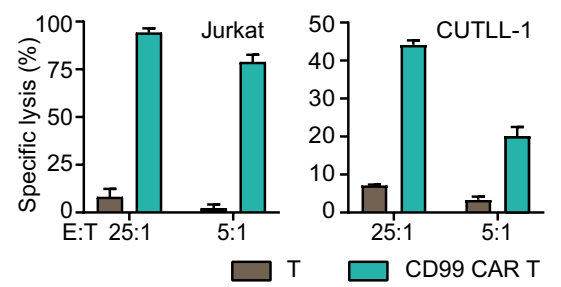

m

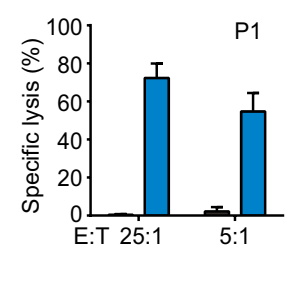

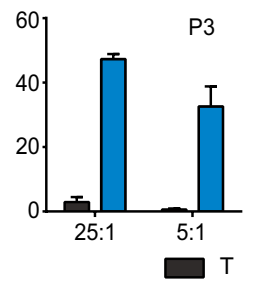

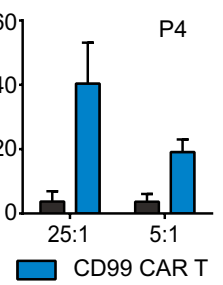

Fig. 1 (See legend on previous page.) 


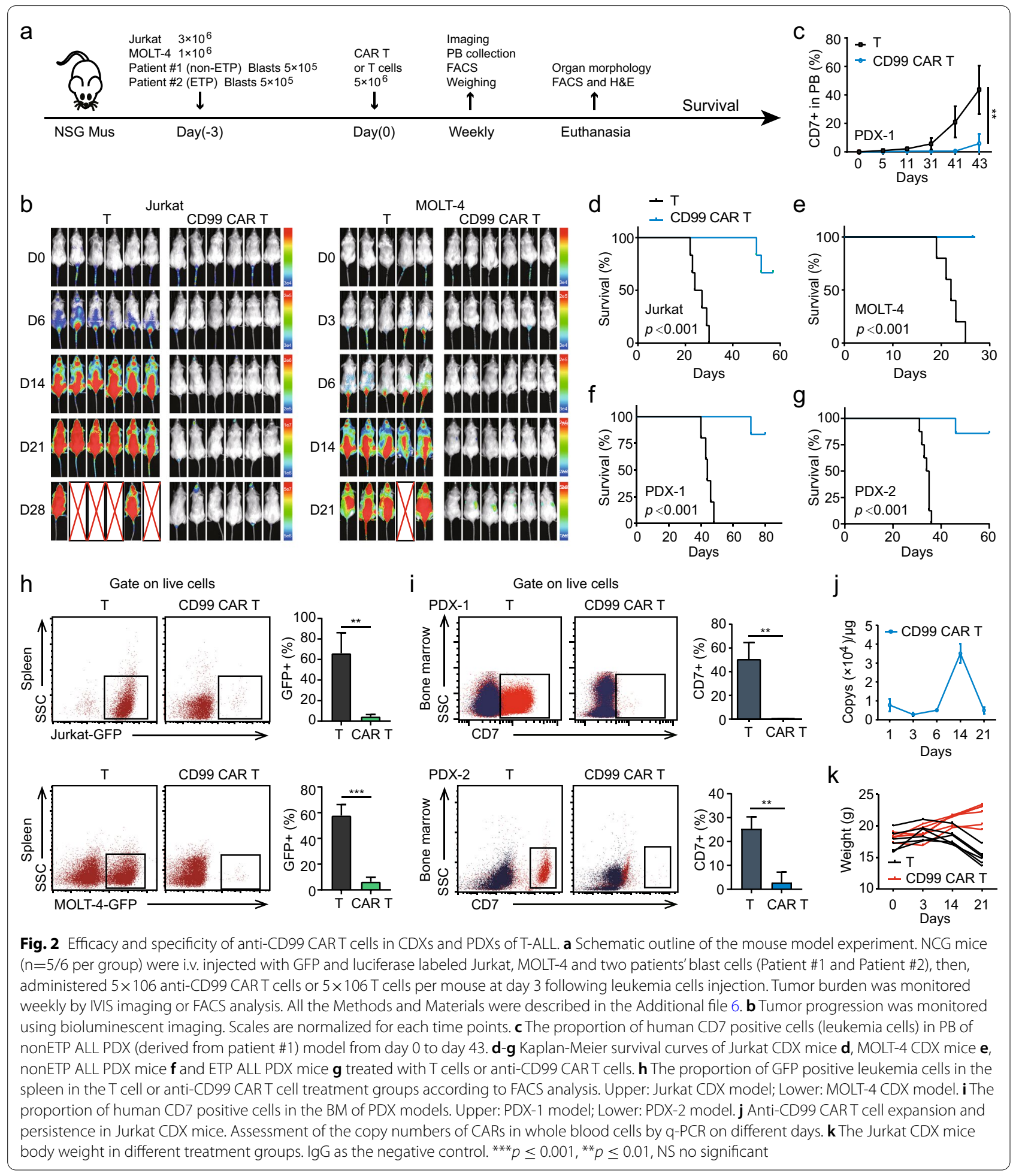

\section{Abbreviations}

T-ALL:T-cell acute lymphoblastic leukemia; ALL: Acute lymphoblastic leukemia; CART: Chimeric antigen receptor T cell; MRD: Minimal residual disease; scFv: Single-chain variable fragment; BM: Bone marrow; AML: Acute myeloid leukemia; CDX: Cell line-derived xenograft; PDX: Patient-derived xenograft; PB: Peripheral blood. 


\section{Supplementary Information}

The online version contains supplementary material available at https://doi. org/10.1186/s13045-021-01178-z.

\begin{abstract}
Additional file 1: Fig. S1. (a) Relative CD99 expression was calculated as CD99 fragments per kilobase of exon model per million mapped fragments (FPKM) on T-ALL samples $(n=264)$ and normal PBMC samples $(n=23)$. Data from Dvinge $\mathrm{H}$ et al. PNAS, 2014 and Liu Y et al. Nature genetic. 2017. (b) The relative CD99 protein level was calculated as the CD99 mean fluorescence intensity (MFI) on T-ALL samples $(n=22)$ and normal T cell samples $(n=5)$ by flow cytometry. (c) Relative CD99 expression was calculated as FPKM on ETP ALL $(n=19)$, nearETP ALL $(n=24)$ and nonETP ALL $(n=146)$ samples. Data from Liu Y et al. Nature genetic. 2017.(d) Relative CD99 expression was calculated as CD99 FPKM on T-ALL subgroup, including LMO2_LYL( $n=18), \operatorname{LMO1} / 2(n=10), \operatorname{HOXA}(n=33), \operatorname{TLX}(n=46), \operatorname{TLX} 1(n=26)$, NKX2_1 $(n=14), \operatorname{TAL} 1(n=87), \operatorname{TAL2}(n=8)$ and unknown $(n=22)$ samples. Data from Data from Liu Y et al. Nature genetic. 2017. (e) Representative immunohistochemistry $(\mathrm{HC})$ images of human normal paraffin tissue sections with the CD99 (12E7) mAb. lgG as the negative control.
\end{abstract}

Additional file 2: Fig. S1. (f) Binding kinetics of anti-CD99 antibodies (1021527 and 3B2/TAB) with CD99 protein. Analysis of the interaction between the antibodies and CD99 protein using BLItz biolayer interferometry. (g) The CD99 expression level in NIH 3 T3 human CD99-overexpression cell line, and anti-CD99 CART cells specifically lysis efficiency at different effector-to-target ratios (1:1/5:1/25:1). (h) The CD99 expression level in MOLT-4 human CD99 knockdown cell line, and anti-CD99 CART cells specifically lysis efficiency at effector-to-target ratios (25:1). (i) The gating strategy of blast cells from T-ALL patients and the CD99 expression level in four patients' blasts (The detail information showed in the Additional file 5: Table S1).

Additional file 3: Fig. S1. (j) Upper: Flow cytometry showing CD99 expression in different AML cell lines. Lower: Cytotoxic activity of antiCD99 CART cells against AML cell lines as determined by calcein release assay at different E:T ratios (1:1/5:1/25:1) after 2-3h of co-culture. (k) Upper: Flow cytometry showing CD99 expression in different solid tumour cell lines. Lower: Cytotoxic activity of anti-CD99 CART cells against various solid tumour cell lines as determined by calcein release assay at different E:T ratios (1:1/5:1/25:1) after 2 -3h of co-culture. ${ }^{* * *} p \leq 0.001,{ }^{* *} p \leq 0.01$, NS $=$ no significant, Scale bar, $50 \mu \mathrm{m}$ or $200 \mu \mathrm{m}$.

Additional file 4: Fig. S2. (a) Spleens from T cell and anti-CD99 CART cell treatment groups were weighed and photographed from the PDX-1. (b) The proportion of human CD7 positive cells in the spleen of PDX-1 models. (c) Spleens from T cell and anti-CD99 CART cell treatment groups were weighed and photographed from the PDX-2. (d) The proportion of human CD7 positive cells in the spleen of PDX-2 models. (e) Histological features of the spleen in the T cell and anti-CD99 CART cell treatment groups

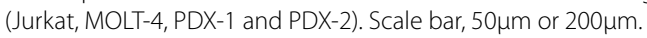

Additional file 5. The patient-related information.

Additional file 6. Methods and Materials.

\section{Acknowledgements}

We thank Peng Zhou, Long Chen and Jian Lin (College of Chemistry and Molecular Engineering, Peking University) for assisting some laboratory experiments. We thank members of our team for critical discussion and suggestions.

\section{Authors' contributions}

$H Z$, JS and TZ designed the experimental plans; JS, ZZ, HC, HW, SZ, YL, AR, XL, $Z Z, X T, Z L$ and $J L$ performed the experiments. JL performed the bioinformatic and statistical analyses; $\mathrm{HZ}$, JS and ZZ analyzed the data and drafted the manuscript. $F Z, Y Q, K Y$ and $H L$ were involved in revision of the manuscript and discussion. All authors read and approved the final manuscript.

\section{Funding}

This work was supported by the grant from the Wuhan Science and Technology Plan Project (2019030703011533) to Tongcun Zhang and also the grant from Postdoctoral Science Foundation of China (2020M682491), Wuhan Former Funded Science and Technology Program (2020020602012111) and grand science and technology special project carried out by the department of Science and Technology of Hubei Province (2020BCB048) to Haichuan Zhu.

\section{Availability of data and materials}

All supporting data are included in the manuscript and supplemental files. Additional data are available upon reasonable request to the corresponding author.

\section{Declarations}

\section{Ethics approval and consent to participate}

This study was approved by the Animal Ethics Committee of Wuhan University of Science and Technology of ID WKD-Zhu-1.

\section{Consent for publication}

Not applicable.

\section{Competing interests}

The authors declare that they have no competing interests.

\section{Author details}

${ }^{1}$ Institute of Biology and Medicine, College of Life and Health Sciences, Wuhan University of Science and Technology, Wuhan 430081, China. ${ }^{2}$ Guangxi Medical University Cancer Hospital, Guangxi 530021, China. ${ }^{3}$ Department of Hematology, Zhongnan Hospital of Wuhan University, Wuhan 430071, China. ${ }^{4}$ Department of Hematology, Tongji Hospital, Tongji Medical College, Hua Zhong University of Science and Technology, Wuhan 430030, China. ${ }^{5}$ Cancer Center, Union Hospital, Tongji Medical College, Huazhong University of Science and Technology, Wuhan 430022, China. ${ }^{6}$ Key Lab of Industrial Fermentation Microbiology of the Ministry of Education and Tianjin Key Lab of Industrial Microbiology, College of Biotechnology, Tianjin University of Science and Technology, Tianjin 300457, China.

Received: 19 Auqust 2021 Accepted: 25 September 2021

Published online: 09 October 2021

\section{References}

1. Belver L, Ferrando A. The genetics and mechanisms of T cell acute lymphoblastic leukaemia. Nat Rev Cancer. 2016;16(8):494.

2. Hunger SP, Mullighan CG. Acute lymphoblastic leukemia in children. N Engl J Med. 2015;373(16):1541-52.

3. Pui C-H, Relling MV, Downing JR. Acute lymphoblastic leukemia. N Engl J Med. 2004;350(15):1535-48.

4. Trinquand A, Tanguy-Schmidt A, Ben Abdelali R, Lambert J, Beldjord K, Lengliné E, De Gunzburg N, Payet-Bornet D, Lhermitte L, Mossafa H. Toward a NOTCH1/FBXW7/RAS/PTEN-based oncogenetic risk classification of adult T-cell acute lymphoblastic leukemia: a Group for Research in Adult Acute Lymphoblastic Leukemia study. J Clin Oncol. 2013;31(34):4333-42.

5. Park JH, Rivière I, Gonen M, Wang X, Sénéchal B, Curran KJ, Sauter C, Wang Y, Santomasso B, Mead E, et al. Long-Term Follow-up of CD19 CAR Therapy in Acute Lymphoblastic Leukemia. N Engl J Med. 2018;378(5):449-59.

6. Maude SL, Laetsch TW, Buechner J, Rives S, Boyer M, Bittencourt H, Bader P, Verneris MR, Stefanski HE, Myers GD, et al. Tisagenlecleucel in Children and Young Adults with B-Cell Lymphoblastic Leukemia. N Engl J Med. 2018;378(5):439-48

7. Alcantara M, Tesio M, June CH, Houot R. CART-cells for T-cell malignancies: challenges in distinguishing between therapeutic, normal, and neoplastic T-cells. Leukemia. 2018;32(11):2307-15.

8. Dworzak MN, Fröschl G, Printz D, Zen LD, Gaipa G, Ratei R, Basso G, Biondi A, Ludwig WD, Gadner H, et al. CD99 expression in T-lineage ALL: implications for flow cytometric detection of minimal residual disease. Leukemia. 2004;18(4):703-8

9. Cox CV, Diamanti P, Moppett JP, Blair AJPO. Investigating CD99 expression in leukemia propagating cells in childhood T cell acute lymphoblastic leukemia. PLOS ONE. 2016;11(10):e0165210.

\section{Publisher's Note}

Springer Nature remains neutral with regard to jurisdictional claims in published maps and institutional affiliations. 